\title{
RELATIVE INTEGRAL BASIS FOR ALGEBRAIC NUMBER FIELDS
}

\author{
MOHMOOD HAGHIGHI \\ Department of Computer Science \\ Bradley University \\ Peoria, Illinois 61625 U.S.A.
}

(Received January 23, 1985 and in revised for March 26, 1985)

ABSTRACI. At first conditions are given for existence of a relative integral basis for $O_{K} \cong O_{k}^{n-1} \oplus 1$ with $[K ; k]=n$. Then the construction of the ideal $I$ in $o_{K} \cong 0_{k}^{n-1} \oplus 1$ is given for proof of existence of a relative integral basis for $\mathrm{O}_{\mathrm{K}_{4}}\left(\sqrt{\mathrm{m}}_{1}, \sqrt{\mathrm{in}_{2}}\right) / \mathrm{O}_{\mathrm{k}}\left({\sqrt{\mathrm{m}_{3}}}_{3}\right)$. Finally existence and construction of the relative integral basis for $0_{k_{6}}\left(\frac{3}{11}, \sqrt{-3}\right) / 0_{k_{3}}(\sqrt[3]{n}), 0_{k_{6}}(\sqrt[3]{11}, \sqrt{-3}) / 0_{k_{2}}(\sqrt{-3})$ for some values of $n$ are given.

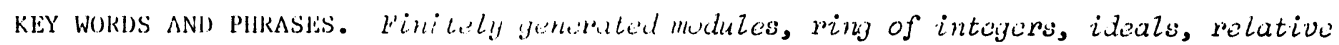
discriminant, class numbers.

1980 AMS SUBEECI CLASSIFICA'ION CODE. Primary 12A30, 12FO5.

1. INTROIJUCTION

Throughout this article the following notation will be used:

Q: $\quad$ field of rational numbers

2: rational integers

$k, K(Q \subseteq k \subseteq K)$ : a]gebraic number $[i e l d s$

disc( $x)$ : discriminant of element $x$

$\mathrm{D}_{\mathrm{K} / \mathrm{k}}$ : relative ditlerent of extension $\mathrm{K} / \mathrm{k}$

$O_{k_{i}}=O_{i} \quad$ ring of integers of $k_{i}$

$h_{k}: \quad c l a s s$ number for $k$

P.I.: principal ideal

$\mathrm{N}_{\mathrm{K} / \mathrm{k}} \mathrm{a}$ : relative norm of an ideal a in $\mathrm{K}$ for extension $\mathrm{K} / \mathrm{k}$.

2. FINITELY GENERATED MOIUUI.ES

In $[1, P .24]$ it was shown that if $M$ is a finitely generated module over a Dedekind $r i n g ~ R$ then

$$
M \cong R^{m} \oplus A \oplus I
$$

where $I$ is an ideal. in $R, \Lambda$ is a torsion-subnodule and $m$ is a positive integer.

Now for extension $k / k$ with $\mid K ; k\}=n$, by (2.1) we have

$$
0_{K} \triangleq 0_{k}^{n-1} \oplus I
$$

so by thiss we have:

THRORLM 2.3. In the extension $k / k$ for $[K ; k]=n$, $o_{K}$ has relative integral basis over $O_{k} \Leftrightarrow I=P \cdot I$. 
IIIIJSTRATIUN 2.4. Let $k_{1}=Q(\sqrt{2}), k_{2}=Q(\sqrt{-7})$. Does a relative integral basis of $0(\sqrt{2}, \sqrt{-7}) / 1)_{3}-0(\sqrt{-14})$ exist? sed al as 121 .

soluthin. By $(2.2)$, a "rolat ive integral basis" exists $\Leftrightarrow \Rightarrow 1=$ P.I., otherwise not.

We will construct in ideal 1 in $o_{k_{3}}$ where $o_{K} \cong o_{k_{3}}^{2-1} \oplus$ I. Since $\left(d_{K_{1}}, d_{K_{2}}\right)=$ $(2 \cdot 4,-7)=1$, then using a theorem given in $[3, p .218]$,

$$
\begin{aligned}
& O_{K}=[1, \sqrt{2}] \times\left[1, \frac{1+\sqrt{-7} \mid}{2} \mid \cdot z=\left[1, \sqrt{2}, \frac{1+\sqrt{-7}}{2}, \frac{\sqrt{2}+\sqrt{-14}}{2}\right] \cdot z,\right. \\
& O_{K}=\left[1, \sqrt{-14}, \frac{1+\sqrt{-7}}{2}, \frac{\sqrt{2}+\sqrt{-14}}{2}\right] \cdot z=[1, \sqrt{-14}] \oplus \mathrm{R},
\end{aligned}
$$

where $R$ is an ${ }_{k}$-module,

$\sqrt{-14} R \subseteq R$

$$
R=\left[\frac{1+\sqrt{-7}}{2}+s+t \sqrt{-14}, \frac{\sqrt{2+\sqrt{-14}}}{2}+u+v \sqrt{-14}\right]
$$

$R=\sqrt{-14} R=\left[\frac{\sqrt{-14}+-7 \sqrt{2}}{2}+s \sqrt{-14}+-14 \mathrm{t},-7+\sqrt{-7}+\mathrm{u} \sqrt{-14}+-14 \mathrm{v}\right]$

We take (2.5) and (2.6) proportional; then

$$
\begin{aligned}
& \left\{\begin{array}{c}
1+\sqrt{-7}+2 s+2 t \sqrt{-14}=-7+v-7+u \sqrt{-14}+-14 \cdot v \\
2 s+2 t \sqrt{-14}=-8+u \sqrt{-14}+-14 v \\
u=2 t, \quad s=-8+-14 v, \text { for } u=v=t=0, s=-4,
\end{array}\right. \\
& \left\{\begin{array}{r}
\sqrt{-14}+-7 \sqrt{2}+2 s \sqrt{-14}-28 t=(\sqrt{2}+\sqrt{-7}+2 u+\sqrt{-14}) \cdot-7 \\
-28 t+\sqrt{-14}(1+2 s)=-14 u+-7 \sqrt{-14}(1+2 v) \\
u=2 t, \quad 1+2 s=-7(1+2 v), \text { for } u=t=v=0, s=-4 .
\end{array}\right.
\end{aligned}
$$

Then,

$$
\begin{aligned}
& R=\left[\frac{1+\sqrt{-7}}{2}-4+0, \frac{\sqrt{2}+\sqrt{-14}}{2}\right]=\left[\frac{-7+\sqrt{-7}}{2}, \frac{\sqrt{2}+\sqrt{-14}}{2}\right] \\
& \sqrt{-14} K=\left[\frac{-7 \sqrt{2}-7 \sqrt{-14}}{2}, \frac{2 \sqrt{-7}-14}{2}\right]=\left[\frac{17(\sqrt{2}+\sqrt{-14}}{2},-7+\sqrt{-7}\right], \\
& R=\frac{\sqrt{-14}(1+\sqrt{-7})}{2 \sqrt{2}} \cdot[2, \sqrt{-14}], \quad K \cong I=[2, \sqrt{-14}] \text { is an ideal in } o_{k}=[1, \sqrt{-14}] \cdot 2 \text {. }
\end{aligned}
$$

Since $1=[2, \sqrt{-14}]$ is not P.l. in $O_{3}$, then $O_{K}$ does not have a relative integral over $0_{3}$. The ideal $1=|2,1-14|$ is unique (up to equivalence of ideals).

The method of the previous theorem is only good for the case $n=2$ since for $n \geq 3$, computiation of an jdeal in $0_{k} \cong 0_{k}^{n-1} \oplus \mathrm{I}$ is too difficult. Thus we need a relation such as the following, between I and one of the invariants in the extension $k / k$.

THEOKIM 2.7. If $C$ is the class of ideals in $k$ containing $d_{K / k}$ and $C_{K / k}$ is a class containing, 1, then

$$
c=c_{k / k}^{2} \text {. }
$$

Now we will give the "criterion for existence of a relative integral basis", for the extension $\mathrm{K} / \mathrm{k}$. See Nurkicwicz et al. $\left[1,4_{4}, 5,6\right]$.

JHEOREM 2.8. Let $[k: k]=n, h_{k}=$ odd, then $o_{K}$ has a "relative integral basis" over $o_{k} \Leftrightarrow d_{k / k}$ (relative discriminant) is a principal ideal. For more details see $[1$, p. 359]. 
PROOF. $\Rightarrow$ : If $O_{k} / O_{k}$ has a relative integral basis, $I=P . I$. Therefore by Theorcim $2.7 \quad d_{k / k}$ is P.I.

$\Leftrightarrow=: \quad d_{K / k}=$ P.I., so every ideal in the class of $c_{K^{\prime} / k}^{2}$ is P.I. Therefore $I^{2}=$ P.I., since $\left(2, h_{k}\right)=1$. Then $l=P .1$. and by $(2.2)$, $O_{K}$ has a relative integral basis over $\mathrm{O}_{\mathrm{k}}$.

COROLLARY 2.9. If $o_{k}=$ P.1.1)., then $h_{k}=1$ is odd and $d_{k / k}=$ P.I. Thus by Theoren 2.8 for every finite extension of $k$ where the ring of integers is its P.I.D., a relative integral basis exists.

IILLSTRATION 2.10. L.et $k_{3}=Q(\sqrt[3]{21})$ and $k=Q(\sqrt{-3}), k_{6}=Q(\sqrt{-3}, \sqrt[3]{2} \sqrt{3}), h_{3}=21$. Does a relative basis of $\mathrm{O}_{\mathrm{K}_{6}} / \mathrm{k}_{3}$ exist or not?

We know that for $n=a b^{2}, \quad(a, b)=1, a b \neq 1$ in $k_{3}=Q(\sqrt[3]{n})$,

$0_{3}=\left[1, \sqrt[3]{\mathrm{ab}^{2}}, \sqrt[3]{\mathrm{a}^{2} \mathrm{~b}}\right] \cdot \%$ for $\mathrm{a} t \pm \mathrm{b} \bmod 9$, and

$0_{3}=\left[\theta_{0}=\frac{1+\sqrt[3]{a b^{2}}+\sqrt[3]{a^{2}} b}{3}, \sqrt[3]{a b^{2}}, \sqrt[3]{a^{2} b}\right] \cdot z$ for $a \equiv b \bmod 9$.

We call these two cases respectively Type I and Type II. $3=(\sqrt{-3})^{2}$ in $k_{2}$. In Type $1,3=3{ }_{11}^{6}, 3{ }_{11}^{2}: 3, \quad 3 \frac{3}{11}=\sqrt{-3}$, so $3=3_{1}^{3}$ and we define $f_{0}=3 a b$. In Type II, $3=3_{11}^{2} 3_{12}^{2} 3_{13}^{2}, 3_{11}^{2}=3_{2}, 3_{12} \cdot 3_{13}=3_{1}, 3_{11} \cdot 3_{12} \cdot 3_{13}=\sqrt{-3}$, so $3=3_{1}^{2} \cdot 3_{2}$, and we dei inc $f_{0}=a b . n_{6 / 3}=3_{11}, d_{6 / 3}=3_{11}^{2}=3_{1}, d_{6 / 3}=3_{1}=$ $(-3, \sqrt[3]{213})$. See $[5, \mathrm{p} \cdot 221]$. By Theorem 2.5 , since $h_{3}$ is odd and $\mathrm{d}_{6 / 3}=(-3, \sqrt[3]{213}-6)=(-343, \sqrt[3]{213}-6)=(\sqrt[3]{213}-6)$ is a P.I., so a relative integral basis exists.

lncidentally in (3.1) wo will prove that if $3 / \mathrm{h}$, then $0_{6}$ has a relative integral basis over $O_{3}$, but here $h_{3}=21$ so $3 / h_{3}$ and also a relative integral basis exists.

3. EXISTENCE OF A RELATNIVE INTLGRAI, BASIS:

BY SONE CONDITIONS ON $n$ FOR $O_{6}(\sqrt[3]{n}, \sqrt{-3}) / \mathrm{O}_{3}(\sqrt[3]{\mathrm{n}})$.

Now here we will show that for some $n \in z$ this extension has relative integral basis. Type 1.

TileORLM 3.1. If $3 / h_{3}$, then $\mathrm{O}_{k_{6}}$ has relative integral basis over $\mathrm{O}_{3}$ for

PROOF. By Theoren in $[7,1,222], 06$ has a relative integral basis over $0_{3} \Leftrightarrow 1_{6 / 3} / \sqrt{-3}=3_{11} / \sqrt{-3}=1 / 3_{1}$ is a $P .1$. in $o_{6}$ generated by an element of $k_{3}$. But $3_{1}=(-3, \sqrt[3]{n}+1)$ when $3 \mid$ ab and $3_{1}=(-3, \sqrt[3]{n})$ when $3 \mid a b$ in Type I and $(3)=\left(3, \cdots 3 \frac{2}{2}\right)$ lior lype 2 .

Now, $3 h_{3}$ so $\left(3_{1}\right)^{3}=(-3, \sqrt[3]{n}+1)^{3}=(3)$ or $\left(3_{1}\right)^{3}=(-3, \sqrt[3]{n})^{3}=(3)$ for P.I., so: $3_{1}=(-3, \sqrt[3]{n}+1)$ or $(-3, \sqrt[3]{n})=3_{1}$ is P.I. Then $1 / 3_{1}$ al so generates a P.I. in $c_{6}$. In Type II, $(3)=\left(3_{1} \cdot 3 \frac{2}{2}\right)$, it is dependent on ideals $3_{1}$ and $3_{2}$; therefore in this case, a relative basis may exist or llay not exist.

But it may be that $3 / h_{3}$ and again $0_{6}$ has relative basis over $0_{3}$. For example in $k_{3}=\left(3(3 / 213), h_{3}=21\right.$ and $3_{1}=(3 / 123-6)$, so $3 \mid 21$ and a relative integral basis exists. Therefore the inverse of Theorem 3.1 is not true in general.

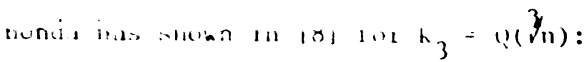


THLOREM 3.2. Oine of the following statements holds:

$$
3\left(h _ { 3 } \Leftrightarrow \left\{\begin{array}{ll}
1) & n=3 \\
2) & n=p, p=p r i m e, p \equiv-1 \quad(\bmod 3) \\
3) & n=3 p \text { or } 9 p, p=p r i m e \equiv 2 \text { or } 5 \text { (mod 9) } \\
4) & n=p \cdot q(p, q \text { are primes), p } \equiv 2 \text { and } q \equiv 5 \text { (mod 9) } \\
5) & n=p^{2} \cdot q \quad(p, q \text { are distinct primes), } p \equiv q \equiv 2 \text { or } 5(\bmod 9) .
\end{array}\right.\right.
$$

DEFINITION 3.3. A number $n$ is called a Honda number if it is a number in the table for theorem 3.2 .

By Theorems 3,1 and 3.2 we have:

THEOREN 3.4. If $\mathrm{n}$ is a llonda number in type $\mathrm{I}, \mathrm{O}_{6}(\sqrt[3]{\mathrm{n}}, \sqrt{-3})$ necessarily has a relative integral basis over $O_{3}(3 / n)$.

4. RELATLE INTESKAL BASIS OF $o_{K_{6}}(\sqrt[3]{n, \sqrt{-3}}) / o_{K_{3}}(\sqrt[3]{n})$.

We proved in lheoreil 3.1 that if $3 / \mathrm{h}_{3}$, then $3_{1}$ is P.I. only for Type $I$. Therefore a relative integral basis for $0_{6} / 0_{3}$ exists, since by the theorem in $\left[3\right.$, p. 201], $\operatorname{disc}\left(1, \frac{\frac{3+\sqrt{-3}}{2}}{3}\right)=\mathrm{d}_{\mathrm{k}_{6} / \mathrm{k}_{3}}=3_{1}$ "Therefore $\left[1, \frac{\frac{3+\sqrt{-3}}{2}}{3_{1}}\right]$ is a relative integral basis for ${ }_{6}$ over $\mathrm{O}_{3}$, so:

$$
0_{k_{6}}=\left[1, \frac{3+\sqrt{-3}}{2}\right] \cdot o_{k_{3}}
$$

5. CONSTRUC'TION OF REIATIVE INTEGRAL, BASIS FOR $0_{6}(\sqrt[3]{\mathrm{n}}, \sqrt{-3}) / 0_{2}(\sqrt{-3})$.

Since $o_{2}(\sqrt{-3})$ is P.1.1)., then by $(2.3) \quad \mathrm{O}_{6}(\sqrt[3]{1,}, \sqrt{-3}) / \mathrm{O}_{2}(\sqrt{-3})$ has a relative intersall bat:is:

$$
\begin{aligned}
& \text { IHLORLM 5.1. Let } \lambda=\frac{3}{\frac{1}{2}(3+\sqrt{-3})} \text {. For Type } I \text { and } 3{ }_{1} \text { is a P.I., if } 3 / a \text { then } \\
& 0_{6}=\left[1, \frac{\sqrt{a b^{2}}}{\lambda}, \frac{\sqrt[3]{a^{2}}}{\lambda^{2}}\right] \cdot 0_{2} \text {, }
\end{aligned}
$$

and if $3 \mid h$ thin

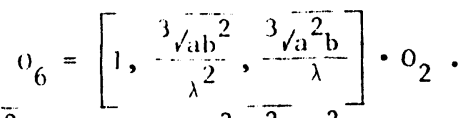

PROOF. Since $\mathrm{N}_{6 / 2}\left({ }^{3} \sqrt{\mathrm{ab}}{ }^{2} / \lambda\right)$ and $\mathrm{N}_{6 / 2}\left({ }^{3} \sqrt{\mathrm{a}{ }^{2} \mathrm{~b} / \lambda^{2}}\right)$ are in $\mathrm{O}_{2}$, then are integers. If $d_{6 / 2}=d i s c\left(1,3, a b^{2} / \lambda, 3 \sqrt{a^{2} b / \lambda^{2}}\right) \cdot 0_{2}$, then by the theorem in [3, p. 201] $x=11, \sqrt[3]{a b})^{2} / \lambda, 3 / a^{2} b / \lambda^{2} \mid$ is a relative integral basis of $0_{6} / 0_{2}$, so we are going to compute disc $(x)$.

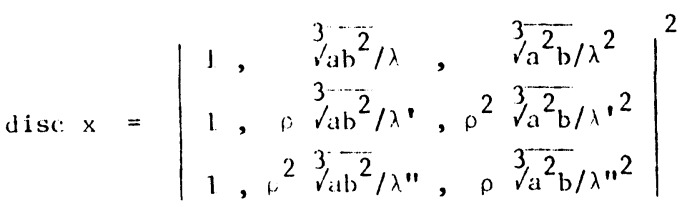

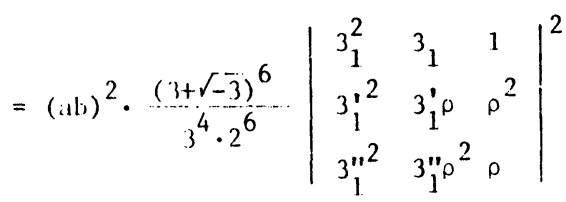




$$
\begin{aligned}
& =\frac{(a b)^{2} \cdot\left(3+1^{\prime}-3\right)^{6}}{3^{4} \cdot 2^{6}}\left\{33_{1}^{2}\left(3 \rho^{2}-3 " \rho\right)+3 i^{2}\left(3 p^{\prime \prime} \rho^{2}-3, \rho\right)+3 p_{1}^{2}\left(3 \rho_{1} \rho^{2}-3 j_{1}^{\prime} \rho\right)\right\}^{2} \\
& =3^{2} \cdot a^{2} b^{2} .
\end{aligned}
$$

For "Iype I" we have $d_{6 / 3}=1_{0}^{2}=$ disc $x$, so $x=\left[1, \sqrt[3 a b]{2} / \lambda, \sqrt[3]{2}{ }^{2} b / \lambda^{2}\right)$ is a relat ive integral bassis: of $06_{6} / 1{ }_{3}$. Sice colm et al. $17,9,101$.

11.I.USTRATron 5.2 . For $k_{3}=q\left(\frac{3}{2} 13\right)$, the ideal $3_{1}=(\sqrt[3]{2} 13-6)$ is P.I. and $3 / a^{2}$ (Type I, 3|a), so

$$
o_{K_{6}}=\left[1, \frac{\sqrt[3]{213}}{\lambda}, \frac{3 / 213^{2}}{\lambda^{2}}\right] \cdot o_{2} \text {, where } \lambda=\frac{\sqrt[3]{213}-6}{\frac{1}{2}(3+\sqrt{-3})} \text {. }
$$

We have Lo mention that it 3 , is not a P.l., this is still an open question.

THEOKLM 5.3. Assiume $k_{3}=\left(0\left(V_{i b} b^{2}\right),(3)=3{ }_{1}^{3}=(\sqrt{a b})^{2} \pm n\right)^{3}$, for 31 ab and "rype [", then:

$$
0_{k_{6}}=\left[1, \frac{3 \cdot 1 b^{2}+11-\sqrt{-3}}{3_{1}}, \frac{3 b^{2}+\sqrt[3]{a^{2} b}+t_{1}}{3_{1}}\right] o_{k_{2}},
$$

where $t_{1}=0$ for $a=3 k+1$ and $b=3 k+2$ or conversely and $t_{1}=1$ for $a=b=3 k+1$ and $\mathrm{t}_{1}=-1$ for $\mathrm{a}=\mathrm{b}=3 \mathrm{k}+2$.

PROOF. NOW

$$
\frac{\sqrt[3]{a b b^{2}}+n-\sqrt{-3}}{3_{1}-u_{1},} \quad \frac{3 a^{2}+\sqrt[3]{a^{2} b}+t_{1}}{3_{1}}=a_{2}
$$

are integ, rals because $\mathrm{N}_{\mathrm{K}_{6}} / \mathrm{K}_{2}\left({ }_{1}\right)$ and $\mathrm{N}_{\mathrm{K}_{6} / \mathrm{K}_{2}}\left(a_{2}\right)$ are integers. We take $\mathrm{x}=\left[1, \alpha_{1}, \alpha_{2}\right]$ and $t_{2}=+n-\sqrt{-3}$ :

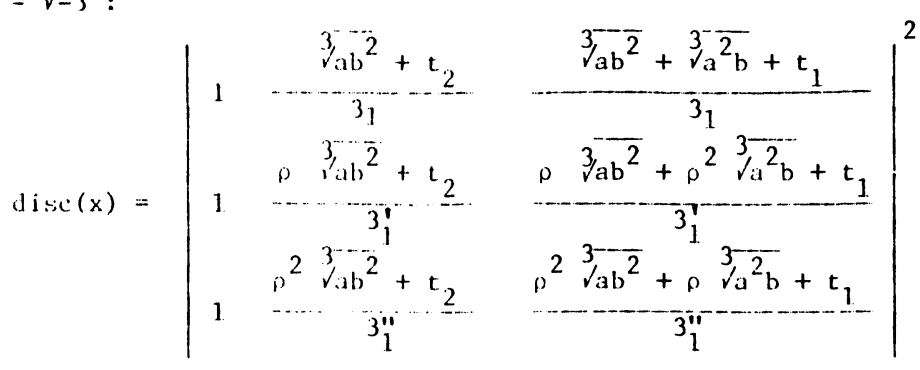

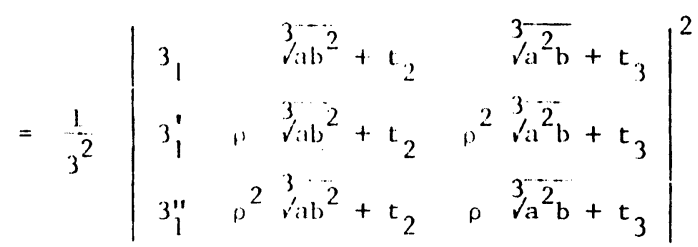

for $t_{3}=t_{1}-t_{2}$.

$$
\begin{aligned}
& \operatorname{disc}(x)=\left[3_{1} \cdot\left(\rho^{2} a b+\rho t_{3} 3 / a b^{2}+t_{2} b^{2} \sqrt[3]{a^{2} b}+t_{2} \cdot t_{3}-\rho a b-\rho^{2} t_{2} \cdot \sqrt[5]{a a^{2} b}-t_{3} p^{2} \sqrt[3]{a b b^{2}}-t_{2} \cdot t_{3}\right)\right. \\
& +3 \frac{1}{1} \cdot\left(\rho^{2} a b+t_{2} \sqrt[3]{a^{2} b}+t_{3} \rho^{2} \sqrt[3]{a b b^{2}}+t_{2} \cdot t_{3}-\rho a b-t_{3} \sqrt[3 a b^{2}]{ }-t_{2} \cdot \sqrt[3]{a^{2} b}-t_{2} \cdot t_{3}\right) \\
& \left.+3 " \cdot \cdot\left(\rho^{2} a b+t_{3} \sqrt[3]{v_{a b}{ }^{2}+t_{2}{ }^{\prime \prime}}{ }^{3} v^{2}{ }^{2} b+t_{2} \cdot t_{3}-\rho a b-t_{2} \sqrt[3]{a^{2} b-\rho t_{3}} \sqrt[3 a b^{2}]{ }+t_{2} \cdot t_{3}\right)\right] \cdot \frac{1}{3^{2}}
\end{aligned}
$$

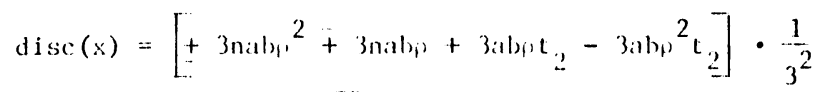

$$
\begin{aligned}
& \text { for } t_{2}=+n-\sqrt{-3} \text { we hive }
\end{aligned}
$$


$\operatorname{disc}(x)=\left[3 n a b\left(p^{2}-\beta\right)-3 a b t_{2}\left(p^{2}-p\right)=3 a b\left(\rho^{2}-\rho\right)(n-t)=3 a b\left(\rho^{2}-\rho\right)(n-n+\sqrt{-3})\right] \cdot \frac{1}{3^{2}}$ $\operatorname{disc}(x)=1 / 3^{2} \cdot 3^{2} \cdot a^{2} b^{2} \cdot 3^{2}$

$\operatorname{disc}(x)=3^{2} \cdot a^{2} b^{2}$, since $d_{k_{6} / k_{2}}=r_{0}^{2}=(3 a b)^{2}=\operatorname{disc}(x)$.

Thus (5.4) is a relative integral bassis for $0_{K_{6}}$ over $0_{k_{2}}$.

Also we have the same result for the case $t_{2}=-n-\sqrt{-3}$.

IIIUSTRATION 5.5. (1) if $k_{3}=\left(!(\sqrt[3]{2}), 3_{1}=(\sqrt[3]{2}+1)\right.$ then $n=1$ and $a=3 k+2$, $b=3 k+1$, so $t_{1}=0$. Therefore:

$$
0_{K_{6}}=\left[1, \frac{3}{2}+\frac{1}{3_{1}}=\sqrt{-3}, \frac{\sqrt[3]{2}+\sqrt[3]{4}+0}{3_{1}}\right] \cdot o_{k_{2}} .
$$

(2) For $k_{3}=2(\sqrt[3]{5}), 3_{1}=(\sqrt[3]{5}-2), \mathrm{n}=-2$ and $t_{1}=0$. We have

$$
o_{k_{6}}=\left[1, \frac{\sqrt[3]{3}-2-\sqrt{-3}}{3_{1}}, \frac{\frac{3}{\sqrt{5}}+\sqrt[3]{25}+0}{3_{1}}\right] \cdot 0_{k_{2}} \text {. }
$$

For all Honda numbers $3_{1}$ is necessarily P.1. so for such $n$ we can construct a relative integral basis as in (5.4) for $\mathrm{O}_{\mathrm{K}_{6}} / \mathrm{K}_{2}$.

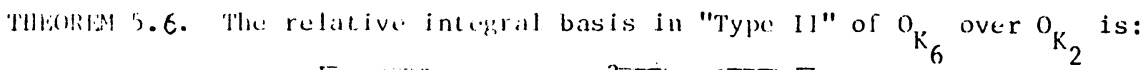

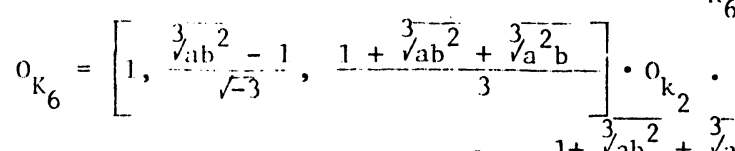

PROOF. For "Type Il" (i.e. a \pm b mod 9 ), $\theta_{0}=\frac{1+\sqrt[3]{\mathrm{ab}^{2}}+\sqrt[3]{\mathrm{a}^{2} \mathrm{~b}}}{3}$ satisfies in

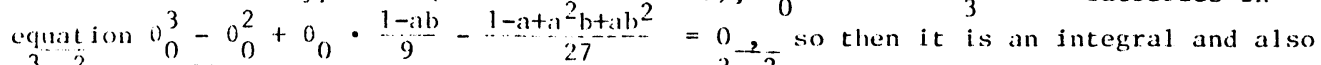
$\left.(\sqrt[3]{a b})^{2}-1\right) / \sqrt{-3}$ is an integrat, because: from $\left(3_{\mathrm{abb}}^{2}-1\right) / \sqrt{-3}=t$, we have $\left(\sqrt[3]{a b^{2}}\right)^{3}=(\sqrt{-3} t+1)^{3}$, so $-3 \sqrt{-3}\left(t^{3}-t\right)=a b^{2}-1+9 t^{2}$ and at $]$ ast we have the equation:

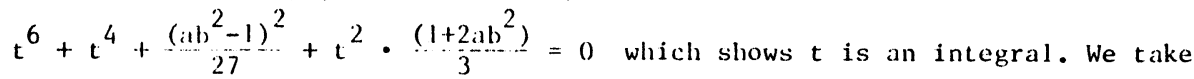
$x=[1, t, 0,1$, then

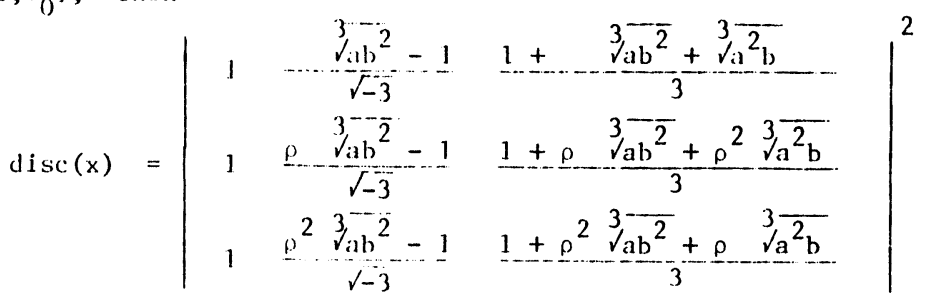

$=\left[\frac{\theta 0}{3 \sqrt{-3}}\left(r^{2} \sqrt[3]{\mathrm{ab}^{2}}-1-\rho \sqrt[3]{a b^{2}}+1\right)+\frac{\theta^{0}}{3 \sqrt{-3}}\left(\sqrt[3]{\mathrm{ab}^{2}}-1-\rho^{2} \sqrt[3]{\mathrm{ab}^{2}}+1\right)+\frac{\theta_{0}^{\prime \prime}}{3 \sqrt{-3}}\left(\rho \sqrt[3 \mathrm{ab}^{2}]{ }-1-\sqrt[3]{\mathrm{ab}^{2}+1}\right)\right]^{2}$ $\operatorname{disc}(x)=\left[\frac{3 a b\left(\rho^{2}-\rho\right)}{3 \sqrt{-3}}\right]^{2}=a^{2} b^{2}$. since $d_{k_{6} / k_{3}}=f_{0}^{2}=(a b)^{2}=\operatorname{disc}(x)$, then $x=\left[1, \frac{\sqrt[3 a b]{ }-1}{\sqrt{-3}}, \frac{\sqrt[3]{a b^{2}}+\sqrt[3]{a^{2}} b+1}{3}\right]_{3}$ is a relative integral basis of $0_{k_{6}} / 0_{k_{2}}$. ILIUSTRATION 5.7. For $k_{3}=Q(3 / 10), a \equiv \pm b \bmod 9$, so

$$
o_{k_{6}}=\left[1, \frac{\sqrt[3]{10}-1}{\sqrt{-3}}, \frac{\sqrt[3]{10}+\sqrt[3]{10^{2}}+1}{3}\right] \cdot o_{k_{2}} \text {. }
$$


llere we will give another theorem for computing a relative integral basis of $\mathrm{O}_{\mathrm{K}_{6}}$ over $\mathrm{O}_{\mathrm{k}_{2}}$ for $\pm \mathrm{n}=3 t+1$ no matter whether $3_{1}$ is a $\mathrm{P}$. I. in $\mathrm{O}_{\mathrm{k}_{3}}$ or not.

THEOREM 5.8. LeL $n=3 t+1, m=-n$ be square-free $i_{n} k_{3}{ }_{3}=Q\left(\begin{array}{c}3 \\ n\end{array}\right)$ for Type $I$, then

or

$$
0_{k_{6}}=\left[\begin{array}{cc}
1+\sqrt[3]{n+\sqrt[3]{n^{2}}} \\
\sqrt{-3}
\end{array}, \frac{3}{\sqrt{n}}, \frac{3-\sqrt{n^{2}}}{3}\right] \cdot 0_{k_{2}},
$$

$$
o_{K_{6}}=\left[\begin{array}{ccc}
1-\sqrt[3]{m+\sqrt[3]{m^{2}}} & 3 \sqrt[3 m]{1-3}, \sqrt[3]{\mathrm{m}^{2}} \\
\sqrt{-3}
\end{array}\right] \cdot \mathrm{o}_{k_{2}} \text {. }
$$

PkOOF. At first we will show that $t=\left(1+\sqrt[3]{n}+\sqrt[3]{n^{2}}\right) / \sqrt{-3}$ is an integral. We: Lake

$$
\begin{aligned}
& t=\frac{(1-\sqrt[3]{n})\left(1+\sqrt[3]{\left.n+3 n^{2}\right)}\right.}{(1+\sqrt[3]{n}) \cdot-\sqrt{-3}}, \quad \frac{(1-n)-t \sqrt{-3}}{\sqrt{-3} t}=\sqrt[3]{n}, \frac{((1-n)-t \sqrt{-3})^{3}}{3 t^{3} \cdot \sqrt{-3}}=n, \\
& (1-n)^{3}+3 t \sqrt[3]{-3}-3+\sqrt{-3}(1-n)^{2}-9 t^{2}(1-n)-n t^{3} \cdot 3 \sqrt{-3}=0, \\
& {\left[\sqrt{-3}\left(3 t^{3}-3 t(1-n)^{2}-3 n t^{3}\right)\right]^{2}=\left[-(1-n)^{3}+9 t^{2}(1-n)\right]^{2},}
\end{aligned}
$$

or brielly:

$$
\begin{gathered}
-27(1-n)^{2} t^{6}-27 t^{4}(1-n)^{2}(2 n+1)-9 t^{2}(1-n)^{4}-(1-n)^{6}=0, \\
t^{6}+(2 n+1) t^{4}+\frac{(1-n)^{2}}{3} \cdot t^{2}+\frac{(1-n)^{4}}{27}=0,
\end{gathered}
$$

which shows that $t$ is an integral. Now we take

$$
x=\left[\frac{1+\sqrt[3]{n+\sqrt[3]{ }}+\frac{3}{\sqrt{n}}, \frac{2}{\sqrt{n}}}{\sqrt{-3}}\right]
$$

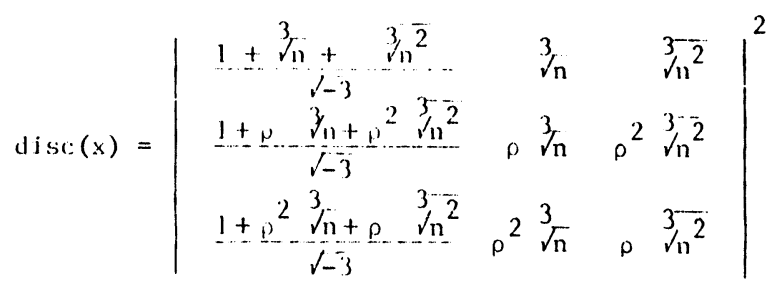

$=n^{2}\left[\frac{1+\sqrt[3]{n}+\sqrt[3]{n^{2}}}{\sqrt{-3}} \cdot\left(\omega^{2}-\rho\right)+\frac{1+\rho \sqrt[3]{n}+\rho^{2} \sqrt[3]{n^{2}}}{\sqrt{-3}} \cdot\left(\rho^{2}-\rho\right)+\frac{1+\rho^{2} \sqrt[3]{n}+\rho \sqrt[3]{n}}{\sqrt{-3}} \cdot\left(\rho^{2}-\rho\right)\right]^{2}$

$=n^{2}\left[\frac{\rho^{2}-\rho}{\sqrt{-3}}(3+0+0)\right]^{2}=3^{2} \cdot n^{2}$

Since $d_{6 / 2}=f_{0}^{2}=(3 n)^{2}=d i s c(x)$, then

$$
o_{K_{6}}=\left[\frac{1+\sqrt[3]{n}+\sqrt[3]{n^{2}}}{\sqrt{-3}}, \frac{3}{\sqrt{n}}, \frac{3}{\sqrt{n^{2}}}\right] \cdot o_{k_{2}} \cdot
$$

We can apply the sillus prowl lin lit $=-11$.

REFERLNCES

1. NARIEWTCZ, W. El ementary and Analytic Theory of Algebraic Numbers, Pwin, Warsaw, 1976.

2. BIRD, R.ll. and PARRY, C... Integral Bases for Bicyclic Biquadratic Fields over Quadratic Subficlds, Palific. J. Math. 66 (1976), 29-36.

3. RiblinulM, P. Algebraic Number Theory, John Wiley \& Sons, New York, 1972.

4. Ak'llN, E. Qnestions de Basse Minimal dans la Theorie Nombres Algebriques, National de la Recherche Scient if ique, XXIV (1950), 19-20. 
5. JoNG, R.L. Stoinitz Classess of Cyclic Extensions of Prime Degree, J. Rejne Angew. M:1th. 250 (1971), 87-98.

6. Sliklik, J.l. Gurp:s locimx, Hermann, Parjs, 1963.

7. CoHN, 11. A Classical luvitat ion to Alfolvaic Numbers and class Field Theory, Springer-Verlas, New York, 1978.

8. HUNDA, T. Pure Cubic liclds whose class Numbers are Multiples of Three, J. Nunber likory 3 (1971), 7-12.

9. BARRUCANI), P. and CUHN, H. Remarks on Principal Factors in a Relative Cubic liclil, I. Numb. Theory 3 (1971), 226-239.

10. (o)tlN, H. Second Course in Number 'lleory, John Wiley, New York, 1962. 


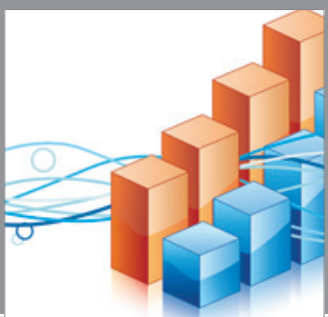

Advances in

Operations Research

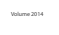

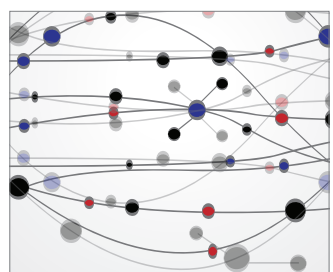

\section{The Scientific} World Journal
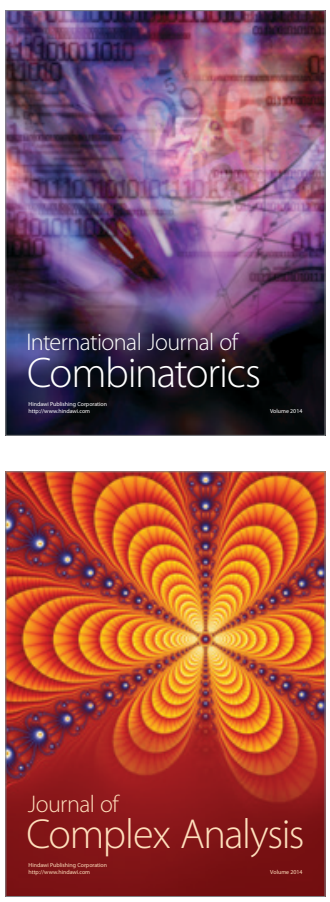

International Journal of

Mathematics and

Mathematical

Sciences
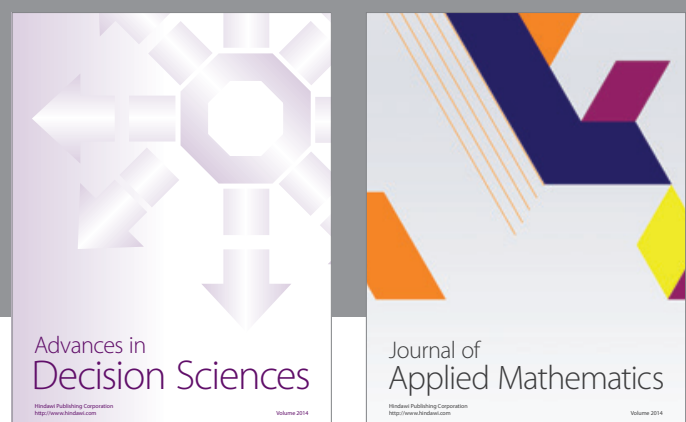

Journal of

Applied Mathematics
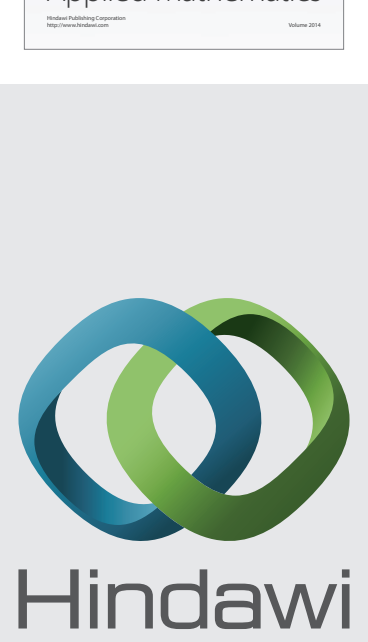

Submit your manuscripts at http://www.hindawi.com
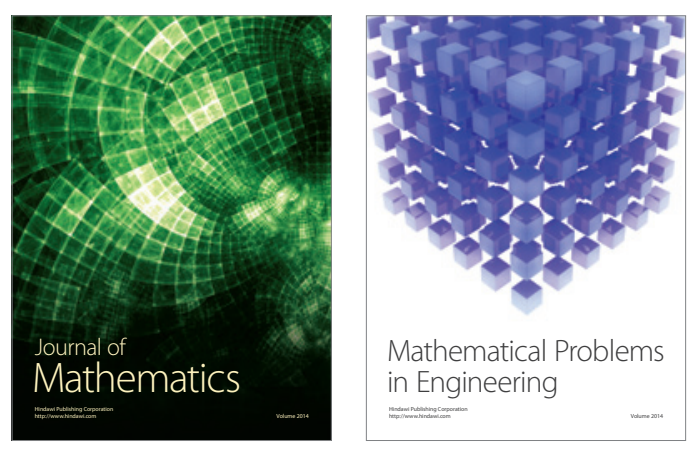

Mathematical Problems in Engineering
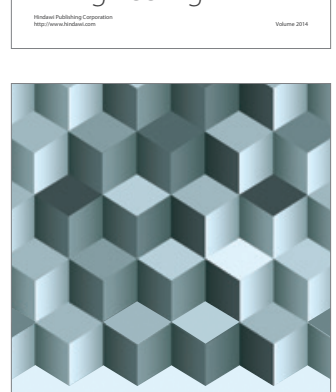

Journal of

Function Spaces
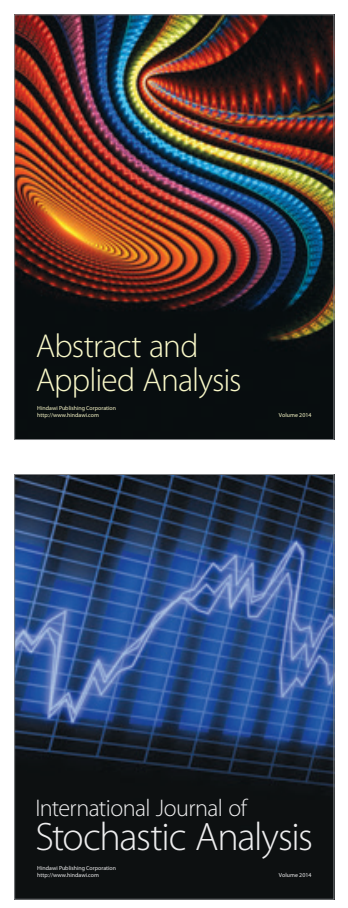

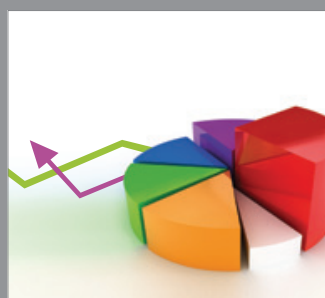

ournal of

Probability and Statistics

Promensencen
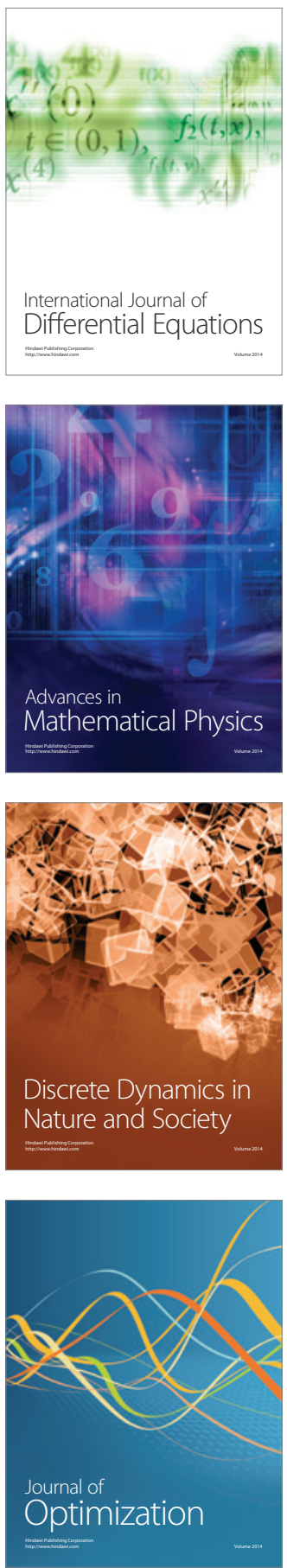